\title{
Unlocking innate healing potentials in patients with type 2 diabetes
}

\author{
Liji Wu $^{1 *}$ and Shao-Nian Yang ${ }^{2 *}$ \\ ${ }^{1}$ Affiliated Hospital of International Mongolian Medicine Hospital of Inner Mongolia, China \\ ${ }^{2}$ The Rolf Luft Research Center for Diabetes and Endocrinology, Karolinska Institutet, Sweden
}

\begin{abstract}
Type 2 diabetes (T2D) is a systemic degenerative disease and progressively changes leading to inconsistent responses to different medications. None of antihyperglycemic drugs even together with other therapies can cure T2D. The human body inherits innate healing potentials to heal diseases including T2D. Healthy lifestyles and alternative medicine can keep innate healing potentials powerful enough to heal T2D by decreasing or abolishing genetic predisposition. Among them, the regulating shoes for self-healing, also called "Chiyu shoes" restore innate healing potentials in T2D patients by combining herbal medicines and magnetic sheets. Such a combination leads to clinical satisfaction in healing T2D.
\end{abstract}

\section{Introduction}

Type 2 diabetes (T2D) is exploding globally, estimated to afflict 415 and 642 million people worldwide in 2015 and 2040, respectively, as shown on the website of International Diabetes Federation. This disease is a life-long condition and needs life-long treatment. It is not only one of the most prevalent diseases, but also one of the most expensive diseases. The global healthcare expenditure to treat T2D is approximated to 850 and 1,000 billion US dollars in 2015 and 2040, respectively, according to information on the Statista website. Currently, antidiabetic therapies mainly focus on glycemic control via administration of insulin and antihyperglycemic drugs [1-3]. These therapies can neither repair the damaged insulin receptor signaling pathway permanently, nor restore functional $\beta$ cell mass efficiently. Therefore, they cannot persistently normalize blood glucose levels to cure diabetes. In addition, they produce numerous adverse effects such as weight gain, hypoglycemia, fluid retention, nausea, vomiting, needle phobia and clinical inertia [1-3]. The huge health and socioeconomic burdens resulting from T2D represent a major challenge to healthcare systems around the world [4,5].

To beat the challenge, type 2 diabetes (T2D) management has radically been rethought [6-8]. It has been realized that the human inherited healing capacity, referred to as "the ability of human body selfhealing" or "innate healing potential", was not paid enough attention to in managing T2D [6-8]. In fact, the healthy body has impressive innate healing potentials to heal diseases. In essence, modification of lifestyles of T2D patients is to promote their innate healing potentials against T2D. Unlocking innate healing potentials holds great hope for defeating T2D. Here we summarize current knowledge on T2D, discuss important roles of innate healing potentials and highlight the novel approach regulating shoes for self-healing, which effectively unlocks innate healing potentials in T2D patients.

\section{A concise picture of type 2 diabetes}

The whole picture of T2D becomes more and more complex. Nevertheless, T2D can succinctly been summarized as a chronic metabolic disorder characterized by hyperglycemia [9-11]. The disease arises from complex interactions between genetic and environmental factors [12-14] (Figure 1). T2D occurs when insulin receptor signaling becomes impaired and/or when pancreatic $\beta$ cells secrete insufficient amounts of insulin $[9,10]$. Pathological alterations in insulin signaling pathways and/or inadequate functional $\beta$ cell mass take center stage in the development of this disease [9-11,15-18]. Concomitantly, many major tissues and organs, including the adipose tissue, skeletal muscle, liver, heart, blood vessels, nerves, eyes and kidneys, undergo obvious pathological changes [9,10,19-22]. Impairments in the adipose tissue, skeletal muscle, liver, brain and microvasculature significantly contribute to aberrant glucose metabolism in T2D [9,10,19-22] (Figure 1). In essence, $T 2 D$ affects an entire human body and is a systemic disease. T2D is also often referred to as a lifestyle disease since adverse lifestyles act as a key driver of T2D, whereas unhealthy inherited traits just make individuals more vulnerable to the disease $[6,14]$.

\section{Innate healing potentials}

The human body inherits an extraordinary capacity to heal itself $[6,8]$. Such an inherited healing capacity, also referred to as innate healing potentials or self-healing ability, is underlain by the dynamic activity of cells, the basic building blocks of the human body. Each cell is constantly checking and regulating its own processes, dynamically

${ }^{\star}$ Correspondence to: Liji Wu, Affiliated Hospital of International Mongolian Medicine Hospital of Inner Mongolia, China, Tel: +8613904711069; E-mail: wulijichiyu@126.com

Shao-Nian Yang, The Rolf Luft Research Center for Diabetes and Endocrinology, Karolinska Institutet, Karolinska University Hospital L1, Sweden, Tel +46851779456; Fax: +46851779450; E-mail: shao-nian.yang@ki.se

Key words: cell regeneration, diabetes, healing potential, hyperglycemia, regulating shoes for self-healing, traditional medicine

Received: February 20, 2020; Accepted: March 04, 2020; Published: March 09, 2020 


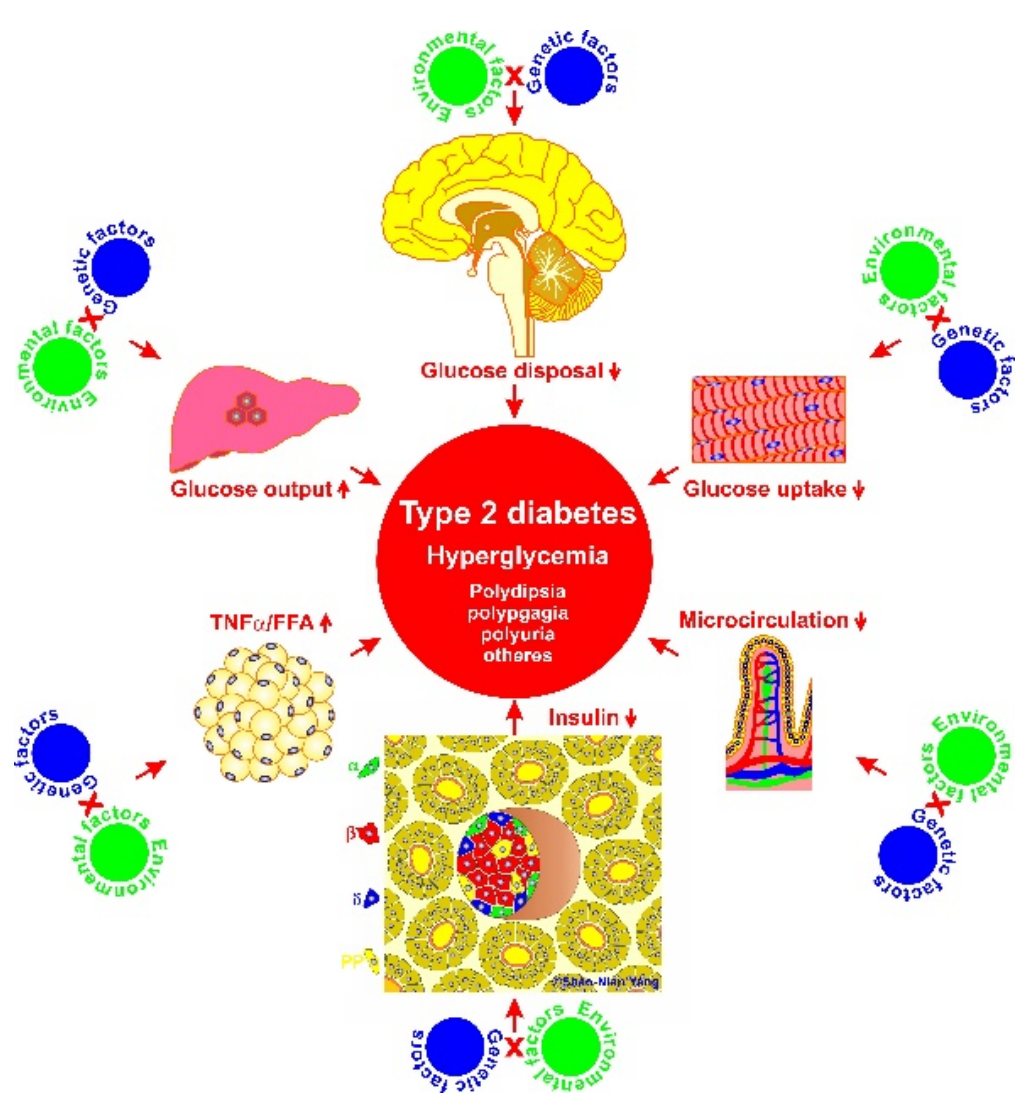

Figure 1. Schematic overview of type 2 diabetes. T2D is caused by complex interactions between genetic and environmental factors. Multiple environmental insults on top of polygenic predisposition result in impaired insulin receptor signaling and/or inadequate functional $\beta$ cell mass. Pathological alterations in insulin signaling pathways and/or inadequate functional $\beta$ cell mass take center stage in the development of this disease. Concomitantly, many major tissues and organs, including the adipose tissue, skeletal muscle, liver, brain and microvasculature, undergo obvious pathological changes resulting in increased plasma tumor necrosis factor $\alpha$ (TNF $\alpha$ )/free fat acids (FFA) from the adipose tissue, decreased glucose uptake by the skeletal muscle, increased glucose output from the liver, decreased glucose disposal mediated by the brain and impaired microcirculation. All these pathological changes contribute hyperglycemia and T2D.

operating to restore itself according to its original genetic blueprint. Some cells can replicate themselves to replace those that have been permanently damaged or destroyed. In addition, adult stem cells and progenitor cells reside in some tissues and organs and differentiate into specific types of cells on-demand. This also participates in formation of innate healing potentials. The innate healing potentials of the human body can alter significantly under pathological scenarios (Figure 2) $[6,8]$. Diseases occur when innate healing potentials are diminished, constrained and locked. Innate healing potentials are modifiable by intervening environmental factors (Figure 2).

Currently, there is no cure for T2D although numerous therapies have been attempted to treat this disease [1-3]. However, the fact that manipulation of environmental factors and in particular lifestyles can effectively prevent and treat T2D convinces us that nature bestows on the human body innate healing potentials to protect against T2D $[6,8,14]$. It is most likely that innate healing potentials in T2D patients are somehow locked up (Figure 2). Unlocking these innate healing potentials should be considered as one of the most promising ways to defeat T2D. World Health Organization encourages people to get rid of drug dependence by maximally taking advantage of innate healing potentials. In many circumstances, innate healing potentials are competent to maintain and restore human health $[6,8]$.

\section{The importance of microcirculation in the maintenance and restoration of innate healing potentials}

Glucose-dependent tissues take up glucose from the blood and oxidize it to generate energy in the form of adenosine triphosphate
(ATP) or to store it in the form of glycogen [23]. Upon entering into cells, glycolysis occurs, thereby producing two pyruvate molecules and a net gain of two ATP molecules from one glucose molecule. After glycolysis, pyruvate moves into the mitochondrial compartment generating additional 34 (in skeletal muscle) or 36 ATP molecules (in other cell types) from two pyruvate molecules [23]. The above two processes occur consecutively under oxygen-sufficient conditions. If there is a lack of oxygen, pyruvate cannot move into mitochondria and just turns into lactic acid [23]. This is why cells including insulinsecreting $\beta$ cells lack energy, undergo dysfunction and even die under hypoxic conditions.

It is well known that long-standing, uncontrolled hyperglycemia brings about functional and structural damage to blood vessels in T2D patients, causing macrocirculatory and microcirculatory complications and aggravating T2D [24,25]. The opposite is also true. Vascular damage, as exemplified in hypertension, is a causal factor for T2D [26]. Undoubtedly, vascular damage is not only a consequence of hyperglycemia, but also can occur prior to T2D as a causal factor for T2D [26]. Vascular damage and in particular microvascular impairment can no longer provide sufficient blood supply to their surrounding cells. Subsequently, hypoxia, nutrient deficiency and metabolic waste accumulation occur, resulting in a series of pathological events [2730]. For example, glucose cannot undergo normal cellular uptake and metabolism under hypoxic conditions [31]. It has suggested that intermittent hypoxia may induce insulin resistance and T2D [30]. $\beta$ cells respond to hypoxia via hypoxia-inducible factor $1 \alpha$ to release less insulin [32]. Recently, a cross-sectional epidemiological study 


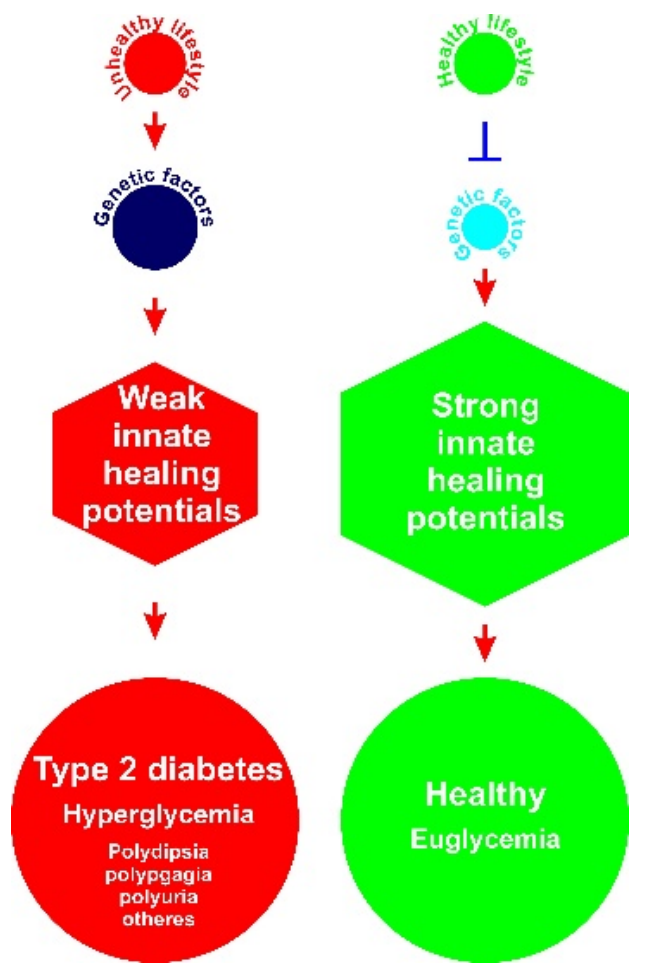

Figure 2. Schematic illustrations of the unlocking of innate healing potentials in patients with type 2 diabetes. Innate healing potentials are negatively and positively regulated by unhealthy and healthy lifestyles, respectively. Unhealthy lifestyles attenuate or lock innate healing potentials to drive the onset of T2D by potentiating genetic predisposition. On the contrary, healthy lifestyles and alternative medicine, such as the regulating shoes for selfhealing, promote innate healing potentials to heal T2D by blocking or at least reducing genetic susceptibility. Therefore, healthy lifestyles and the regulating shoes for self-healing can unlock innate healing potentials in T2D patients to help them defeat the disease.

verifies the association between hypoxia and glucose intolerance in people living at high altitudes in the Tibetan highland [29]. Current knowledge pinpoints microcirculatory impairment as a matter of the utmost importance in the maintenance and restoration of innate healing potentials to prevent and heal T2D.

\section{A new development in unlocking of innate healing po- tentials in patients with type 2 diabetes}

In recent years, many new technologies have been emerging in both modern and traditional disciplines [33,34]. Clinical Medicine including T2D treatments have benefited greatly from these new technologies. Here we focus on the regulating shoes for self-healing, also called "Chiyu Shoes" [35].

\section{General aspects of the regulating shoes for self-healing}

Herbal medicines have massively been benefiting T2D patients at least in some parts of the world, such as China and India. Mr. Wu has invented an ingenious method to restore innate healing potentials in patients with T2D by combining herbal medicines and magnetic sheets in specially designed walking shoes, namely, the regulating shoes for selfhealing [35]. Four and eleven invention patents for the regulating shoes for self-healing have been filed in Europe and China, respectively. Thirty-five certificates of copyrighted works based on the regulating shoes for selfhealing in treatment of geriatric diseases have been issued.

According to therapeutic roles of these Mongolian herbal medicines documented in ancient medicinal writings, bioeffects of magnetic fields and recent clinical observations, the regulating shoes for self- healing act as an effective therapeutic tool against hyperglycemia and hypertension most likely through complex mechanisms. Sixty-eight magnet sheets in a regulating shoe for self-healing are just placed in close proximity to the skin of the foot to produce optimal magnetic fields above blood flow through the foot. The magnetic fields act directly on the primary endogenous magnetic target hemoglobin in the blood because hemoglobin is an iron-containing protein [36]. This results in an elevation of the deoxygenation rate of hemoglobin and a reduction of blood viscosity $[37,38]$. Subchronic exposure to magnetic fields also increases red blood cell counts and hemoglobin levels in the body [39]. Moreover, magnetic fields also regulate some signaling pathways, such as the nitric oxide pathway [40-44]. All these biological effects can promote blood flow, oxygen and nutrient supply to and metabolic waste removal from cells, tissues and organs throughout the body. Thereby, they expedite functional restoration and morphological regeneration of various cells and tissues including insulin-secreting $\beta$ cells, insulin receptor-expressing cells and glucose-dependent tissues. In fact, blood gas measurements have visualized increased oxygen levels in the blood of wearers of the regulating shoes for self-healing.

On the other hand, 12 types of Mongolian antidiabetic herbal medicines in each Energy Box of the regulating shoes for self-healing produce a variety of additive and synergistic effects against the disease [45]. These effects range from ameliorating insulin-, insulin signalingand $\beta$ cell mass-related impairments to normalizing glucose metabolism [45]. These herbal medicines simultaneously and synergistically act on different pathogenic mechanisms of T2D resulting in satisfactory healing in T2D patients [45].

In addition, the magnetic fields and herbal medicines also collaboratively interplay with each other to optimize their antidiabetic roles. Wearing the regulating shoes for self-healing ameliorates microcirculatory perturbations not only in the foot, but also in many major tissues and organs throughout the body [35]. Wearing the regulating shoes for self-healing 10 hours a day for three months has been demonstrated to effectively normalize blood glucose and blood pressure levels in T2D patients [35]. Indeed, the therapy with the regulating shoes for self-healing has yielded impressive clinical outcomes in T2D patients [35]. This emphasizes that the complex systemic disease T2D involved in multiple pathogenic mechanisms cannot be cured by symptomic treatment against hyperglycemia alone but can be satisfactorily healed by releasing constrained innate healing potentials in T2D patients. This is convincingly exemplified by therapy with the regulating shoes for self-healing as an effective and innovative form of complementary and alternative medicine in T2D management.

\section{Historical overview of the regulating shoes for self-healing}

The regulating shoes for self-healing began to enter design development phase in 1993. A clinical study on the shoes was carried out in China International Mongolian Medical Hospital of Inner Mongolia and Inner Mongolia Autonomous Region's Hospital in 1995. This clinical study demonstrates that the total effective rate was $90.1 \%$ in T2D patients wearing the regulating shoes for self-healing. Moreover, these patients had completely dropped any other antidiabetic therapy in the clinical period [35].

Subsequently, another clinical research on the regulating shoes for self-healing was implemented in China International Mongolian Medical Hospital of Inner Mongolia, Affiliated Hospital of Inner Mongolia Medical University in 2010. Similarly, 92\% T2D patient was effectively treated by wearing the regulating shoes for self-healing and did not suffer adverse effects [35]. 
Recently, a new clinical trial was performed in Institute of Traditional Medicine and Technology of Mongolia, the First State Central Hospital of Mongolia, the Second State Central Hospital, the Third State Central Hospital and Bayanzurkh Hospital for Diabetes during the period from November 2015 to June 2016. Among a total of 269 patients with diabetes, 57, 95, 130, 158, 200 and 249 patients became normal glycemic (about $6 \mathrm{mM}$ ) after they wore the regulating shoes for self-healing for up to $30,60,90,120,150$ and 180 days, respectively. The average blood glucose level in these 249 patients was decreased to $5.96 \mathrm{mM}$. These effectively treated patients account for $92.57 \%$ of all patients included in this clinical trial. Except these 249 patients, another 20 patients with a serious medical condition had a significant decrease in their blood glucose values from 17.89 to $7.27 \mathrm{mM}$. A total of $87.98 \%$ of patients did not require insulin and hypoglycemic agents anymore when they wore the regulating shoes for self-healing for up to 180 days.

\section{Conclusions}

Unsuccessful attempts to cure T2D have provoked reconsideration of strategies in T2D management. As a matter of fact, the human body possesses innate healing potentials to heal diseases including T2D. Innate healing potentials are negatively regulated by genetic and environmental risk factors (Figure 2). Adverse environmental risk factors diminish, constrain or lock innate healing potentials to provoke the onset of T2D by potentiating the diabetic effects of T2D loci (Figure 2). On the contrary, healthy lifestyles can keep innate healing potentials strong enough to heal T2D by blocking or at least reducing the diabetic effects of T2D loci (Figure 2). Therefore, diet control, physical exercise, weight loss, smoking cessation, drinking in moderation, mind-body exercise and herbal medicines can facilitate unlocking of innate healing potentials in T2D patients to help them defeat the disease.

New ideas and technologies emerged in both modern and traditional disciplines have resulted in some novel smart options to combat T2D, such as the regulating shoes for self-healing [35]. The regulating shoes for self-healing restores innate healing potentials in T2D patients by combining herbal medicines and magnetic sheets in a walking shoe [35]. Such a combination of herbal medicines and magnetic sheets collaboratively acts on various pathogenic mechanisms of T2D leading to clinical satisfaction in healing T2D.

\section{References}

1. Tahrani AA, Barnett AH, Bailey CJ (2016) Pharmacology and therapeutic implications of current drugs for type 2 diabetes mellitus. Nat Rev Endocrinol 12: 566-592.

2. Nathan DM (2015) Diabetes: Advances in diagnosis and treatment. JAMA 314: 1052 1062.

3. Inzucchi SE, Majumdar SK (2016) Current therapies for the medical management of diabetes. Obstet Gynecol 127: 780-794.

4. Alberti KG, Zimmet PZ (2014) Diabetes: a look to the future. Lancet Diabetes Endocrinol 2: e1-e2.

5. Zimmet PZ, Magliano DJ, Herman WH, Shaw JE (2014) Diabetes: a 21st century challenge. Lancet Diabetes Endocrinol 2: 56-64.

6. Kligler B (2004) The role of the optimal healing environment in the care of patients with diabetes mellitus type II. J Altern Complement Med 10: S223-S229.

7. Sakallaris BR, MacAllister L, Voss M, Smith K, Jonas WB (2015) Optimal healing environments. Glob Adv Health Med 4: 40-45.

8. O’Donnell M (2004) Health-promotion behaviors that promote self-healing. J Altern Complement Med 10: S49-S60.

9. American Diabetes Association (2014) Diagnosis and classification of diabetes mellitus. Diabetes Care 37: S81-S90.
10. Inzucchi SE (2012) Clinical practice. Diagnosis of diabetes. N Engl J Med 367: 542550 .

11. Yang SN, Berggren PO (2019) The eye as a novel imaging site in diabetes research Pharmacol Ther 197: 103-121.

12. Bell GI, Polonsky KS (2001) Diabetes mellitus and genetically programmed defects in beta-cell function. Nature 414: 788-791.

13. Owen KR, McCarthy MI (2007) Genetics of type 2 diabetes. Curr Opin Genet Dev 17: 239-244.

14. Cornelis MC, Hu FB (2012) Gene-environment interactions in the development of type 2 diabetes: recent progress and continuing challenges. Annu Rev Nutr 32: 245-259.

15. Yang SN, Berggren PO (2005) $\beta-$ Cell $\mathrm{Ca}_{\mathrm{v}}$ channel regulation in physiology and pathophysiology. Am J Physiol Endocrinol Metab 288: E16-E28.

16. Yang SN, Berggren PO (2005) $\mathrm{Ca}_{\mathrm{v}} 2.3$ channel and $\mathrm{PKC} \lambda$ : new players in insulin secretion. J Clin Invest 115: 16-20.

17. Yang SN, Berggren PO (2006) The role of voltage-gated calcium channels in pancreatic $\beta$-cell physiology and pathophysiology. Endocr Rev 27: 621-676.

18. Yang SN, Shi Y, Yang G, Li Y, Yu J, et al. (2014) Ionic mechanisms in pancreatic $\beta$-cell signaling. Cell Mol Life Sci 71: 4149-4177.

19. Schwartz MW, Seeley RJ, Tschop MH, Woods SC, Morton GJ, et al. (2013) Cooperation between brain and islet in glucose homeostasis and diabetes. Nature 503: 59-66.

20. Guilherme A, Virbasius JV, Puri V, Czech MP (2008) Adipocyte dysfunctions linking obesity to insulin resistance and type 2 diabetes. Nat Rev Mol Cell Biol 9: 367-377.

21. Heni M, Kullmann S, Preissl H, Fritsche A, Haring HU (2015) Impaired insulin action in the human brain: causes and metabolic consequences. Nat Rev Endocrinol 11: 701-711.

22. DeFronzo RA, Tripathy D (2009) Skeletal muscle insulin resistance is the primary defect in type 2 diabetes. Diabetes Care 32: S157-S163.

23. Lecka-Czernik B, Rosen CJ (2015) Energy excess, glucose utilization, and skeletal remodeling: new insights. J Bone Miner Res 30: 1356-1361.

24. Forbes JM, Cooper ME (2013) Mechanisms of diabetic complications. Physiol Rev 93: 137-188.

25. Harcourt BE, Penfold SA, Forbes JM (2013) Coming full circle in diabetes mellitus: from complications to initiation. Nat Rev Endocrinol 9: 113-123.

26. Emdin CA, Anderson SG, Woodward M, Rahimi K (2015) Usual blood pressure and risk of new-onset diabetes: Evidence from 4.1 million adults and a meta-analysis of prospective studies. J Am Coll Cardiol 66: 1552-1562.

27. Narimiya M, Yamada H, Matsuba I, Ikeda YU, Tanese T, et al. (1982) The effect of hypoxia on insulin and glucagon secretion in the perfused pancreas of the rat. Endocrinology 111: 1010-1014.

28. Dionne KE, Colton CK, Yarmush ML (1993) Effect of hypoxia on insulin secretion by isolated rat and canine islets of Langerhans. Diabetes 42: 12-21.

29. Okumiya K, Sakamoto R, Ishimoto Y, Kimura Y, Fukutomi E, et al. (2016) Glucose intolerance associated with hypoxia in people living at high altitudes in the Tibetan highland. BMJ Open 6: e009728.

30. Rasouli N (2016) Adipose tissue hypoxia and insulin resistance. J Investig Med 64 830-832.

31. Xi L, Chow CM, Kong X (2016) Role of tissue and systemic hypoxia in obesity and type 2 diabetes. J Diabetes Res 2016: 1527852.

32. Miao G, Ostrowski RP, Mace J, Hough J, Hopper A, et al. (2006) Dynamic production of hypoxia-inducible factor-1alpha in early transplanted islets. Am J Transplant 6 : 2636-2643.

33. Wall D, Ray W, Pathak RD, Lin SM (2014) A google glass application to support shoppers with dietary management of diabetes. J Diabetes Sci Technol 8: 1245-1246.

34. Garabedian LF, Ross-Degnan D, Wharam JF (2015) Mobile phone and smartphone technologies for diabetes care and self-management. Curr Diab Rep 15: 109.

35. Wu L (2016) Repairing the ability of human body self-healing--Diabetes can be selfhealed. World Latest Medicine Information 16: 166-167.

36. Mattison SP, Kim W, Park J, Applegate BE (2014) Molecular imaging in optical coherence tomography. Curr Mol Imaging 3: 88-105. 
37. Muehsam D, Lalezari P, Lekhraj R, Abruzzo PM, Bolotta A, et al. (2013) Non-thermal radio frequency and static magnetic fields increase rate of hemoglobin deoxygenation in a cell-free preparation. PLoS One 8: e61752.

38. Tao R, Huang K (2011) Reducing blood viscosity with magnetic fields. Phys Rev E Stat Nonlin Soft Matter Phys 84: 011905.

39. Salem A, Hafedh A, Rached A, Mohsen S, Khemais BR (2005) Zinc prevents hematological and biochemical alterations induced by static magnetic field in rats. Pharmacol Rep 57: 616-622.

40. Pilla A, Fitzsimmons R, Muehsam D, Wu J, Rohde C, et al. (2011) Electromagnetic fields as first messenger in biological signaling: Application to calmodulin-dependent signaling in tissue repair. Biochim Biophys Acta 1810: 1236-1245.

41. Pilla AA (2012) Electromagnetic fields instantaneously modulate nitric oxide signaling in challenged biological systems. Biochem Biophys Res Commun 426: 330-333.
42. Diniz P, Soejima K, Ito G (2002) Nitric oxide mediates the effects of pulsed electromagnetic field stimulation on the osteoblast proliferation and differentiation. Nitric Oxide 7: 18-23.

43. Cheng G, Zhai Y, Chen K, Zhou J, Han G, et al. (2011) Sinusoidal electromagnetic field stimulates rat osteoblast differentiation and maturation via activation of NO-cGMPPKG pathway. Nitric Oxide 25: 316-325.

44. Fitzsimmons RJ, Gordon SL, Kronberg J, Ganey T, Pilla AA (2008) A pulsing electric field (PEF) increases human chondrocyte proliferation through a transduction pathway involving nitric oxide signaling. J Orthop Res 26: 854-859.

45. Zhang HM, Liang FX, Chen R (2015) Ancient records and modern research on the mechanisms of Chinese herbal medicines in the treatment of diabetes mellitus. Evid Based Complement Alternat Med 2015: 747982.

Copyright: $\odot 2020 \mathrm{Wu} \mathrm{L}$. This is an open-access article distributed under the terms of the Creative Commons Attribution License, which permits unrestricted use, distribution, and reproduction in any medium, provided the original author and source are credited. 